\title{
Long noncoding RNA KCNQ1OT1 contributes to tumor growth and activates Wnt/ $\beta$-catenin signaling in osteosarcoma by targeting the miR-3666/KLF7 axis
}

\author{
AIJUN HUANG ${ }^{1}$, SONG JIN ${ }^{1}$, WEIDONG HAN ${ }^{2}$, YIMING WANG ${ }^{1}$, SHUQIANG MA ${ }^{1}$, ZENGRONG WANG ${ }^{1}$, \\ KUN LIN $^{1}$, QINGBAO ZOU ${ }^{1}$, JINGYING ZHOU ${ }^{1}$, ZHONGTAN LI ${ }^{1}$ and LIPING CHEN ${ }^{3}$ \\ Departments of ${ }^{1}$ Orthopaedic Surgery and ${ }^{2}$ Pain Medicine, The Eighth Affiliated Hospital, \\ Sun Yat-Sen University, Shenzhen, Guangdong 518033; ${ }^{3}$ Department of Nephrology, \\ The Second People's Hospital of Futian District, Shenzhen, Guangdong 518049, P.R. China
}

Received July 23, 2019; Accepted June 18, 2020

DOI: $10.3892 / \mathrm{ijmm} .2020 .4768$

\begin{abstract}
Osteosarcoma (OS) is a type of primary malignant cancer occurring in the bone and poses a threat to the lives of children and young adults. Long non-coding RNAs (lncRNAs) have been certified to play important roles in various human malignant tumors, including OS. IncRNA KCNQ1OT1 has been investigated in certain types of cancer; however, its role and molecular mechanisms in OS remain to be determined. In the present study, a high KCNQ1OT1 expression was detected in human OS tissues and cell lines. Moreover, patients with OS with a high expression of KCNQ1OT1 presented a worse prognosis. Loss-of-function assays demonstrated that KCNQ1OT1 silencing suppressed cell proliferative, migratory and invasive abilities in OS. Importantly, the knockdown of KCNQ1OT1 suppressed the $\mathrm{Wnt} / \beta$-catenin signaling pathway in OS. In vivo assays displayed the inhibitory role of the silencing of KCNQ1OT1 in OS tumor growth. As regards the underlying mechanisms, KCNQ1OT1 could sponge miR-3666, and its expression was negatively associated with that of miR-3666 in OS tissues. Thereafter, Kruppel-like factor 7 (KLF7), upregulated in OS tissues and cells, was discerned as a target gene of miR-3666. Furthermore, KLF7 expression negatively correlated with miR-3666 expression, whereas it positively correlated with KCNQ1OT1 expression. A rescue assay delineated that the overexpression of KLF7 counteracted the KCNQ1OT1 knockdown-induced suppression of OS cell proliferation, migration, invasion and $\mathrm{Wnt} / \beta$-catenin signaling. Collectively, the present study demonstrates that KCNQ1OT1 facilitates
\end{abstract}

Correspondence to: Dr Liping Chen, Department of Nephrology, The Second People's Hospital of Futian District, 27 Zhongkang Road, Shenzhen, Guangdong 518049, P.R. China

E-mail: 410616707@qq.com

Key words: KCNQ1OT1, miR-3666, Kruppel-like factor 7, Wnt/ $\beta$-catenin signaling, osteosarcoma
OS progression and activates $\mathrm{Wnt} / \beta$-catenin signaling by targeting the miR-3666/KLF7 axis.

\section{Introduction}

Osteosarcoma (OS) is a type of malignant bone tumor which predominantly occurs in adolescents $(1,2)$. As one of the aggressive types of cancer, OS is associated with a high risk of mortality. Over the past years, OS has been effectively treated by complete surgical resection (3). With the deep understanding of OS progression in recent years, neoadjuvant and adjuvant chemotherapy have been applied to the treatment of patients with OS $(4,5)$. Despite great advances being made in OS treatment, the prognosis of patients with OS remains unsatisfactory due to distant metastasis $(6,7)$. Therefore, it is essential to further explore the potential molecular mechanisms underlying OS progression in order to improve the prognosis of patients with OS.

Long non-coding RNAs (lncRNAs) are a group of RNAs with $>200$ nucleotides in length. Due to lack of a complete open reading frame, they are limited to encode proteins (8). It has been demonstrated that lncRNAs, aberrantly expressed in cancers, can regulate various biological processes (9). For example, LINC00163 was found at a low level in lung cancer and a high LINC00163 expression predicted a better prognosis (10). IncRNA CRNDE has been shown to serve as a tumor promoter and to promote cell proliferation, migration and invasion in non-small cell lung cancer (NSCLC) via sponging miR-338-3p (11). LncRNA XIST was abnormally expressed in esophageal cancer and promoted cancer development via sponging miR-494 and targeting CDK6 (12). According to previous studies, IncRNA KCNQ1OT1 was discovered to be an oncogene in multiple types of cancer, such as colorectal cancer, NSCLC and colon cancer (13-15). Nevertheless, the functional role and mechanisms of action of KCNQ1OT1 in OS warrant further investigation.

$\mathrm{Wnt} / \beta$-catenin signaling has been reported in various types of cancer and plays pivotal role in cancer development and progression. For example, LINC00675 promotes cell proliferation, migration and invasion via activating 
Wnt/ $\beta$-catenin signaling in cervical cancer (16). LINC01606 has been shown to be associated with Wnt/ $\beta$-catenin signaling, and to promoted metastasis and invasion in gastric cancer (17). However, research on the association between KCNQ1OT1 and $\mathrm{Wnt} / \beta$-catenin signaling has not been conducted in OS to date, at least to the best of our knowledge.

The present study focused on investigating the function of KCNQ1OT1 in OS. The results validated that KCNQ1OT1 predicted a poor prognosis, facilitated OS cell proliferative, migratory and invasive abilities, and activated $\mathrm{Wnt} / \beta$-catenin signaling by targeting the miR-3666/KLF7 axis, which suggests that KCNQ1OT1 may serve as a prognostic biomarker in OS.

\section{Materials and methods}

Tissue samples. A total of 40 pairs of OS tissues and matched normal adjacent tissues were collected from the Eighth Affiliated Hospital, Sun Yat-Sen University from November, 2013 to December, 2018. All patients provided written informed consent and the approval of this study was obtained from the Ethics Committee of the Eighth Affiliated Hospital, Sun Yat-Sen University.

Cells and cell culture. Three human OS cell lines (U2OS, SaoS-2 and MG-63) and an osteoblast cell line (hFOB) were purchased from Cell Bank of the Chinese Academy of Science. The cell lines were cultured in DMEM (Gibco; Thermo Fisher Scientific, Inc.) in a humidified $5 \% \mathrm{CO}_{2}$ atmosphere at $37^{\circ} \mathrm{C}$ supplemented with $10 \%$ fetal bovine serum (Invitrogen; Thermo Fisher Scientific, Inc.), $100 \mu \mathrm{g} / \mathrm{ml}$ penicillin and $100 \mu \mathrm{g} / \mathrm{ml}$ streptomycin (Invitrogen; Thermo Fisher Scientific, Inc.).

Cell transfection. For transfection, short hairpin RNA (shRNA; $50 \mathrm{nM}$ ) plasmids directly targeting KCNQ1OT1 (sh-KCNQ1OT1\#1/2/3), the negative control (sh-NC; $50 \mathrm{nM})$, miR-3666 mimics $(30 \mathrm{nM})$ and the negative control (NC mimics; $30 \mathrm{nM}$ ) were all respectively synthesized by GenePharma. The sequences were as follows: 5'-CTTCAA CCCTTAGGTACAACACCAAAAC-3' (sh-KCNQ1OT1\#1), 5'-ACCAAGACTCAGTCCCGGGCTTAATCCT-3' (sh-KCN Q1OT1\#2), 5'-TCCTAGCCCTCAGACTCAACCCCTGG AC-3' (sh-KCNQ1OT1\#3), 5'-CAAGCTTAACAGAGAGAC CAAAAGAACA-3' (sh-NC), 5'-CAGTGCAAGTGTAGA TGCCGAGTCACGTTCACATCTACGGCT-3' (miR-3666 mimics), 5'-AGACAAGGACAGATCGAAAAGTCTGTT CCTGTCTAGCTTTTC-3' (NC mimics). For KLF7 overexpression, the full-length of KLF7 cDNA was amplified and inserted into pcDNA3.1. An empty vector pcDNA3.1 (30 nM, Invitrogen; Thermo Fisher Scientific, Inc.) was served as a negative control. Lipofectamine 3000 reagent (Invitrogen; Thermo Fisher Scientific, Inc.) was applied to conduct all transfections for $48 \mathrm{~h}$ and cells were collected for subsequent experiments at 3-4 passages.

Reverse transcription-quantitative PCR (RT-qPCR). Total RNA was isolated from tumor tissues and transfected cells using TRIzol reagent (Invitrogen; Thermo Fisher Scientific, Inc.). Total RNA was transcribed into cDNA using the cDNA
Reverse Transcription kit (Applied Biological Materials Inc.). The miR-3666 level was examined using the TaqMan MicroRNA Assays kit (Applied Biosystems; Thermo Fisher Scientific,Inc.). qPCR was performed using the 7300 Real Time PCR System (Applied Biosystems; Thermo Fisher Scientific, Inc.). The PCR primers were as followings: GAPDH forward, 5'-GGGAGAAGCTGAGTCATGGG-3' and reverse, 5'-TCC CGGTGACATTTACAGCC-3'; U6 forward, 5'-CGCGATATG GTTTTGGCAGG-3' and reverse, 5'-TGGACGTATTCGATC AGCCG-3'; miR-3666 forward, 5'-TGGGATGGATTGCAG GTTGAA-3' and reverse, 5'-GCAGAGGCTTCTTCCTCA TGT-3'; KCNQ1OT1 forward, 5'-GGGTCTAGGGTCCAC ATCCT-3' and reverse, 5'-AGACTCCCGATCCTCTGTCC-3'; KLF7 forward, 5'-TGTAGGCAGAACAAGCGGG-3' and reverse, 5'-ATGTGGCCACTTGTGAGAGC-3'. The reaction conditions comprised of a pre-denaturation at $95^{\circ} \mathrm{C}$ for $10 \mathrm{~min}$, with a total of 40 cycles of denaturation at $95^{\circ} \mathrm{C}$ for $15 \mathrm{sec}$, annealing at an appropriate annealing temperature for $1 \mathrm{~min}$, and extension at $72^{\circ} \mathrm{C}$ for $30 \mathrm{sec}$. All values were normalized to GAPDH or U6, and all data were calculated using the $2^{-\Delta \Delta \mathrm{Cq}}$ method (18).

Cell Counting kit-8 (CCK8) assay. Cells were plated in a 96-well plate at a density of $2 \times 10^{4}$ cells/well and $10 \mu \mathrm{l}$ of CCK-8 reagent was added to each well daily. Following incubation for $2 \mathrm{~h}$ at $37^{\circ} \mathrm{C}$, microplate reader (Bio-Rad Laboratories, Inc.) was employed to examine the absorbance value of each well at a wavelength of $450 \mathrm{~nm}$.

Colony formation assay. Cells (500 per well) transfected with sh-KCNQ1OT1 were seeded in a 6-well plate. Following incubation at $37^{\circ} \mathrm{C}$ in $5 \% \mathrm{CO}_{2}$, cell colonies were fixed with 4\% paraformaldehyde for 10 min (Sigma Aldrich) and then stained with $0.1 \%$ crystal violet for $5 \mathrm{~min}$ at room temperature (Sigma-Aldrich; Merck KGaA). A microscope was then used to count colonies (Leica MZ8, Leica Microsystems GmbH).

Transwell assay. The assessment of cell migration or invasion was conducted by the use of Transwell chambers (Corning Costar, Inc.) without or with Matrigel. A total of $2 \times 10^{4}$ cells were starved in serum-free DMEM medium and then placed into the upper chamber. DMEM medium with $20 \%$ serum was placed into lower chamber. Following incubation for $48 \mathrm{~h}$ at $37^{\circ} \mathrm{C}$, the migrated or invaded cells in the lower chamber were fixed with 4\% paraformaldehyde (Sigma Aldrich; Merck $\mathrm{KGaA}$ ) for $15 \mathrm{~min}$ and stained with $0.5 \%$ crystal violet for $15 \mathrm{~min}$ at room temperature (C0121, Beyotime Institute of Biotechnology), respectively. Finally, the cells were counted under a light microscope (x200 magnification, Olympus Corporation).

Xenograft tumor model. A total of 6 male 4-week-old BALB/c nude mice were available from the Eighth Affiliated Hospital, Sun Yat-Sen University and housed in the animal laboratory with laminar flow equipment under special pathogen-free (SPF) conditions and $12 \mathrm{~h}$ light-dark cycles. The temperature was set at $18-22^{\circ} \mathrm{C}$ and the relative humidity was $50-60 \%$, and the animals had free access to autoclaved food and water during the study. The animal-related protocol was approved by the Animal Care and Experiment Committee of the Eighth 
Affiliated Hospital, Sun Yat-Sen University. U2OS cells transfected with sh-KCNQ1OT1\#1 or sh-NC were injected subcutaneously into the mice ( $\mathrm{n}=3$ per group) for 28 -day inoculation purposes, with the health of the mice and tumor growth monitored every 4 days. When mice exhibited tumor metastasis, lethargy, weight loss in excess of $20 \%$ body weight or other signs of distress consistent with the IACUC standards, they were sacrificed by cervical dislocation. Mice were verified dead by observing respiration, heartbeat, pupils and nerve reflex. Tumors were then excised carefully from the mice and weighed for analysis. Tumor volume was estimated using digital calipers and calculated using the following the formula: $0.5 \mathrm{x}$ length $\mathrm{x}$ width ${ }^{2}$.

Luciferase reporter assay. The KCNQ1OT1-WT/KLF7-WT vector was constructed by cloning KCNQ1OT1/3'untranslated region (3'-UTR) of KLF7 covering the miR-3666 binding site into the pmirGLO reporter vector (Promega Corp.). The vector KCNQ1OT1-Mut/KLF7-Mut was inserted by the mutant KCNQ1OT1/KLF7. miR-3666 mimics or NC mimics were then respectively co-transfected with KCNQ1OT1-WT/KLF7-WT or KCNQ1OT1-Mut/KLF7-Mut. All transfections were performed with the use of Lipofectamine 3000 (Invitrogen; Thermo Fisher Scientific, Inc.). After 48 h later, the Dual Luciferase Reporter Assay System (Promega Corp.) was used to measure the luciferase activity and normalized to the activity of the Renilla luciferase gene.

Nuclear-cytoplasmic fractionation. The nuclear and cytoplasmic fractions were separated with a PARIS kit (Invitrogen; Thermo Fisher Scientific, Inc.). Briefly, cells were re-suspended in cell fraction buffer and incubated on ice for $10 \mathrm{~min}$. The cytoplasmic fraction and nuclear pellet were collected following centrifugation at $500 \mathrm{x} \mathrm{g}$ for $5 \mathrm{~min}$ at $4^{\circ} \mathrm{C}$. Subsequently, KCNQ1OT1 expression was evaluated by RT-qPCR with GAPDH as cytoplasmic control and U6 as nuclear control.

Bioinformatics analysis. The potential bindings between miRNAsand KCNQ1OT1 were predicted by starBase (http://starbase.sysu.edu.cn/). Online tools of RNA22 (https://cm.jefferson. edu/rna22/Interactive/), PicTar (https://pictar.mdc-berlin.de/), TargetScan (http://www.targetscan.org/vert_72/) and microT (http://diana.imis.athena-innovation.gr/DianaTools/index.php?r= microT_CDS/index), were used to predict the target gene of miR-3666.

Western blot analysis. RIPA lysis buffer was applied to lyse the cells and thereby the proteins were collected. Protein concentrations were measured using the BCA Protein Assay kit (Pierce; Thermo Fisher Scientific, Inc.). Proteins were loaded on $10 \%$ SDS-PAGE with $20 \mu \mathrm{g}$ protein per lane and transferred onto PVDF membranes. Subsequently, 5\% skimmed milk powder was employed to block the PVDF membranes for $2 \mathrm{~h}$, and the membranes were incubated with primary antibodies to $\beta$-catenin (1:8,000, ab32572, Abcam), c-Myc (1:1,000, ab166837, Abcam), matrix metalloproteinase (MMP)7 (1:1,000, ab207299, Abcam) and GAPDH (1:2,000, ab8245, Abcam) overnight at $4^{\circ} \mathrm{C}$. The membranes were then incubated with goat anti-mouse $\operatorname{IgG} \mathrm{H} \& \mathrm{~L}$ conjugated to HRP $(1: 8,000 ; \mathrm{ab} 97040, \mathrm{Abcam})$ for $2 \mathrm{~h}$ at room temperature.
Finally, the immunofluorescence of protein bands was visualized with ECL detection system (Pierce; Thermo Fisher Scientific, Inc.). The Bio-Rad image analysis system (Bio-Rad Laboratories, Inc.) was used to obtain images, and Quantity One version 4.6.2 software (Bio-Rad Laboratories, Inc.) was used for analysis. The relative protein level was expressed by the gray value of the corresponding protein band value of the GAPDH protein band.

RIP assay. RIP assay was carried out using the EZ Magna RNA immunoprecipitation kit (EMD Millipore). Briefly, RIP lysis buffer was used to lyse the OS cells. The cell lysates were then cultivated with magnetic beads conjugated with specific antibodies to human Ago2 (1:2,000, ab186733, Abcam) or IgG $\left(1: 2,000\right.$, ab190475, Abcam) at $4^{\circ} \mathrm{C}$ for $3 \mathrm{~h}$ until overnight. Finally, the immunoprecipitates were purified and subjected to RT-qPCR.

Statistical analysis. Statistical analyses were conducted with SPSS 21.0 statistical software (SPSS Inc.). Data are expressed as the means \pm SD and each experiment was conducted in triplicate. The differences among groups were detected by one-way or two-way ANOVA followed by Tukey's post-hoc test or the Student's t-test. Pearson's correlation analysis was conducted to determine the correlation between the expression of miR-3666 and KCNQ1OT1 or KLF7 in OS tissues. An overall survival curve was drawn to determine survival analysis using the Kaplan-Meier method and log-rank test. Data were also analyzed by Pearson's $\chi^{2}$ test. A P-value $<0.05$ was considered to indicate a statistically significant difference.

\section{Results}

Overexpression of KCNQ1OT1 accelerates OS progression and activates Wnt/ $\beta$-catenin signaling. To determine the expression pattern of KCNQ1OT1 in OS, KCNQ1OT1 expression was detected in OS tissues and adjacent non-tumor tissues. The results of RT-qPCR revealed that KCNQ1OT1 exhibited a higher level in OS tissues than in adjacent normal tissues. Consistently, a high KCNQ1OT1 expression was found in OS cell lines, particularly in the U2OS and SaoS-2 cells (Fig. 1A). Through Kaplan-Meier survival analysis, it was concluded that patients with OS with a high KCNQ1OT1 expression exhibited a relatively low overall survival compared to those with a low KCNQ1OT1 expression (Fig. S1). In addition, it was found that KCNQ1OT1 expression was not associated with age or sex, but was associated with tumor size and TNM stage (Table I). These results indicated the potential oncogenic role of KCNQ1OT1 in OS. T

To further explore biological function of KCNQ1OT1 in OS, a loss-of-function assay was conducted. Firstly, the expression of KCNQ1OT1 was stably silenced in the U2OS and SaoS-2 cells by transfection with sh-KCNQ1OT1\#1/2/3 (Fig. 1B). Owing to the optimal transfection efficiency, sh-KCNQ1OT1\#1 and sh-KCNQ1OT1\#2 were selected for use in subsequent experiments. Through CCK- 8 and colony formation assays, it was observed that the deficiency of KCNQ1OT1 overtly suppressed the proliferation and colony formation ability of the U2OS and SaoS-2 cells (Fig. 1C and D). Transwell assay exhibited that OS cell 

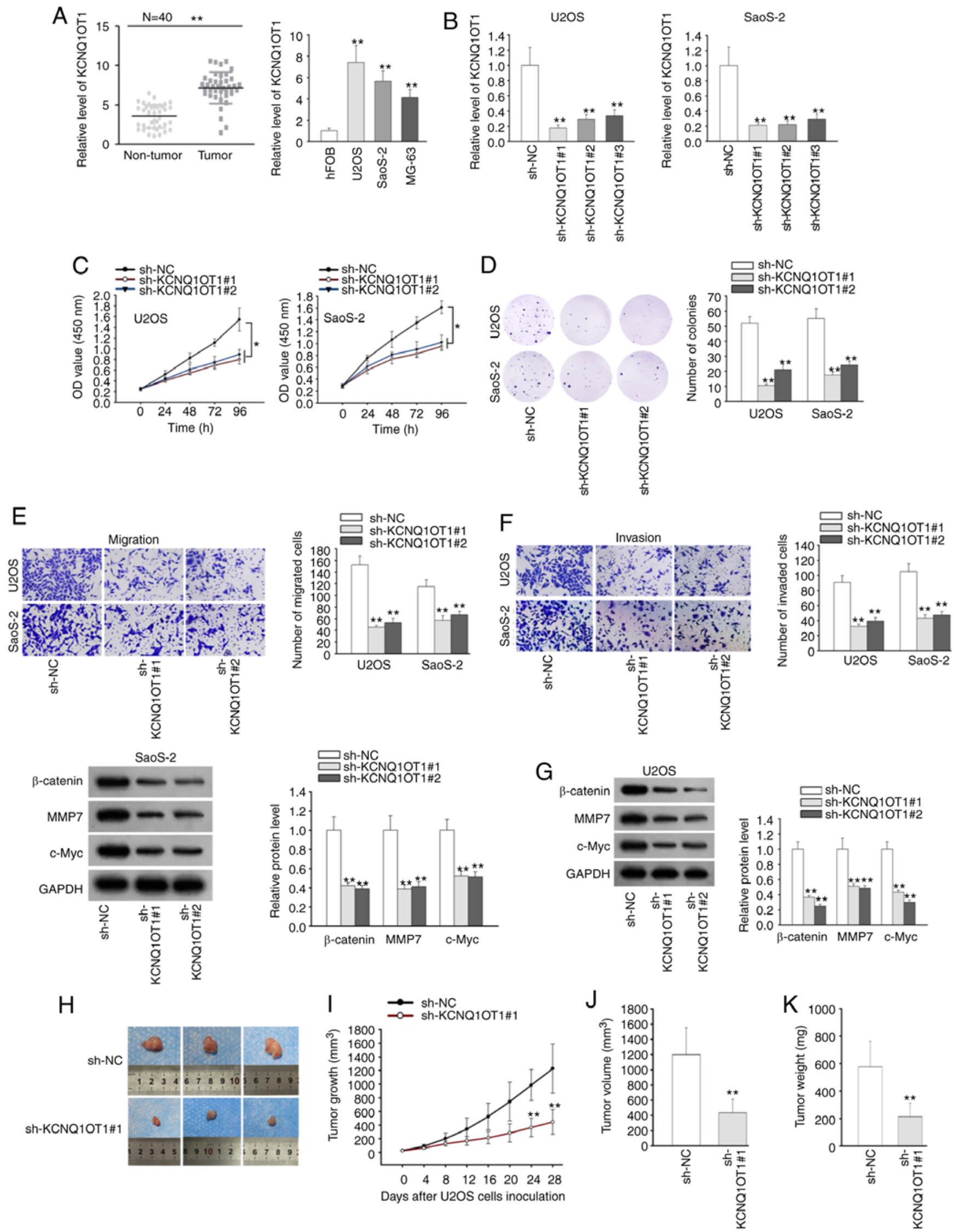

Figure 1. Overexpression of KCNQ1OT1 accelerates OS progression and activates Wnt/ $\beta$-catenin signaling. (A) KCNQ1OT1 expression in OS tissues and cell lines was detected by RT-qPCR. (B) RT-qPCR manifested the knockdown efficiency of sh-KCNQ1OT1\#1/2/3 in OS cells. (C and D) CCK-8 and colony formation assays delineated the proliferation of sh-KCNQ1OT1\#1/2-transfected OS cells. (E and F) The migratory and invasive capacities of cells transfected with sh-KCNQ1OT1\#1/2 were tested by transwell assay. (G) Western blot analysis disclosed protein levels of $\beta$-catenin, MMP7 and c-Myc upon KCNQ1OT1 knockdown. (H) Images of xenograft tumors excised from mice in sh-KCNQ1OT1\#1 group and sh-NC group. (I and J) Tumor growth curve and tumor volume in the sh-KCNQ1OT1\#1 group and sh-NC group are shown. (K) Tumor weight in sh-KCNQ1OT1\#1 group and sh-NC group is presented. ${ }^{*} \mathrm{P}<0.05,{ }^{* *} \mathrm{P}<0.01$ vs. respective control. OS, osteosarcoma. 
Table I. Association between KCNQ1OT1 expression and clinical features of patients with OS $(n=40)$.

\begin{tabular}{lrrr}
\hline & \multicolumn{2}{c}{$\begin{array}{c}\text { KCNQ1OT1 } \\
\text { expression }\end{array}$} & \\
\cline { 2 - 3 } Variable & Low & High & P-value \\
\hline Age, years & & & \\
$\quad<60$ & 12 & 14 & 0.7410 \\
$\geq 60$ & 8 & 6 & \\
Sex & & & \\
$\quad$ Male & 13 & 11 & 0.7475 \\
Female & 7 & 9 & \\
Tumor size, cm & & & \\
$\leq 5$ & 15 & 3 & 0.0003 \\
$>5$ & 5 & 17 & \\
TNM stage & & & 0.0079 \\
I-II & 12 & 3 & \\
III-IV & 8 & 17 & \\
\hline
\end{tabular}

Low/high expression determined by the sample median. Data were analyzed by Pearson's $\chi^{2}$ test. $\mathrm{P}<0.05$ was considered to indicate a statistically significant difference.

migration and invasion were considerably suppressed upon KCNQ1OT1 silencing (Fig. 1E and F). Of note, KCNQ1OT1 was previously reported to promote osteogenic differentiation by activating Wnt/ $\beta$-catenin signaling (19). Therefore, the present study investigated whether KCNQ1OT1 exerts an effect on Wnt/ $\beta$-catenin signaling in OS. Through western blot analysis, it was found that the protein levels of Wnt/ $\beta$-catenin signaling downstream genes ( $\beta$-catenin, MMP7 and c-Myc) were significantly decreased in the sh-KCNQ1OT1\#1/2-transfected OS cells (Fig. 1G), which suggested that KCNQ1OT1 activated Wnt/ $\beta$-catenin signaling in OS. Subsequently, the role of KCNQ1OT1 in tumor growth was further investigated by using an in vivo assay. In this experiment, each nude mouse was burdened with one tumor, and the size of the tumors in the sh-KCNQ1OT1\#1 group was smaller than that of those in the sh-NC group (Fig. 1H). Moreover, it was revealed that sh-KCNQ1OT1\#1 transfection suppressed tumor growth and volume (Fig. 1I and J). The maximum diameter of tumors obtained was $17 \mathrm{~mm}$ and the maximum volume was $1,224 \mathrm{~mm}^{3}$. In addition, the weight of tumors was found to be decreased in the sh-KCNQ1OT1\#1 group compared with the sh-NC group (Fig. 1K). All these data indicated that KCNQ1OT1 was overexpressed, and promoted cellular progression and activated Wnt/ $\beta$-catenin signaling in OS.

KCNQ1OT1 serves as a molecular sponge of miR-3666. Subsequently, the underlying mechanisms of KCNQ1OT1 in OS were investigated. It has been widely reported that lncRNAs can function competing endogenous RNAs (ceRNAs) by competitively interacting with miRNAs to release their target mRNAs in the cytoplasm $(20,21)$. Thus, the present study assessed the distribution of KCNQ1OT1 in OS cells via nuclear-cytoplasmic fractionation assay. The results revealed that KCNQ1OT1 was mainly distributed in the cytoplasm of OS cells (Fig. 2A), indicating the ceRNA hypothesis. Using starBase (http://starbase.sysu.edu.cn/), several miRNAs possessing binding sites for KCNQ1OT1 were predicted (Fig. 2B). RT-qPCR analysis revealed that KCNQ1OT1 silencing markedly increased the expression of miR-3666, whereas it did not affect the expression of other miRNAs (Fig. 2C). Thus, miR-3666 was selected for analysis. The binding site between miR-3666 and KCNQ1OT1 is illustrated in Fig. 2D. Subsequently, miR-3666 expression in OS tissues and cells was evaluated, and the results demonstrated that miR-3666 expression was downregulated in both OS tissues and cell lines (Fig. 2E). Pearson's correlation analysis further validated the negative correlation between the expression of KCNQ1OT1 and miR-3666 in OS tissues (Fig. 2F). Luciferase reporter assay revealed that the overexpression of miR-3666 caused an overt decrease in the luciferase activity of the KCNQ1OT1-WT reporter, while that of the KCNQ1OT1-Mut reporter remained unaltered (Fig. 2G). The results from RIP assay also revealed the abundant enrichment of KCNQ1OT1 and miR-3666 in the anti-Ago2 group compared with anti-IgG group (Fig. 2H). From the above-mentioned findings, it was thus concluded that KCNQ1OT1 can sponge miR-3666 in OS cells.

KLF7 is a downstream target of miR-3666. Subsequently, the downstream target of miR-3666 was further investigated. Combining the online tools of RNA22, PicTar, TargetScan and microT, KLF7 was predicted to be the target gene of miR-3666 (Fig. 3A). As shown in Fig. 3B, miR-3666 contained a binding site on KLF7 3'UTR. Thereby, the expression of KLF7 in OS tissues and cells was analyzed by RT-qPCR, and the results revealed the high expression of KLF7 in OS tissues and cells (Fig. 3C and D). Furthermore, the expression of KLF7 was found to negatively correlate with miR-3666 expression, and to positively correlate with KCNQ1OT1 expression by Pearson's correlation analysis (Fig. 3E). Luciferase reporter assay disclosed that miR-3666 overexpression reduced the luciferase activity of the KLF7-WT reporter, but exhibited no effect on the KLF7-Mut reporter (Fig. 3F). On the basis of RIP assay, the anti-Ago2 group rather than the anti-IgG group exhibited a notable enrichment of KCNQ1OT1, miR-3666 and KLF7 (Fig. 3G). Taken together, these findings indicate that miR-3666 can bind to KLF7 in OS cells.

KCNQ1OT1 promotes OS progression and activates Wnt/ $\beta$-catenin signaling by targeting the $m i R-3666 / K L F 7$ axis. Finally, the effect of the KCNQ1OT1/miR-3666/KLF7 axis on OS progression was verified. KLF7 was firstly overexpressed in U2OS cells, and the expression of KLF7 was markedly increased by pcDNA3.1/KLF7 transfection (Fig. 4A). CCK-8 and colony formation assays revealed that the inhibitory effects of KCNQ1OT1 suppression or miR-3666 overexpression on OS cell proliferation were reversed by the upregulation of KLF7 (Figs. 4B and C and S2A and B). Based on Transwell assay, KLF7 overexpression recovered the impaired migration and invasion induced by the silencing of KCNQ1OT1 or overexpression of miR-3666 (Figs. 4D and E and S2C and D). Western blot analysis revealed 

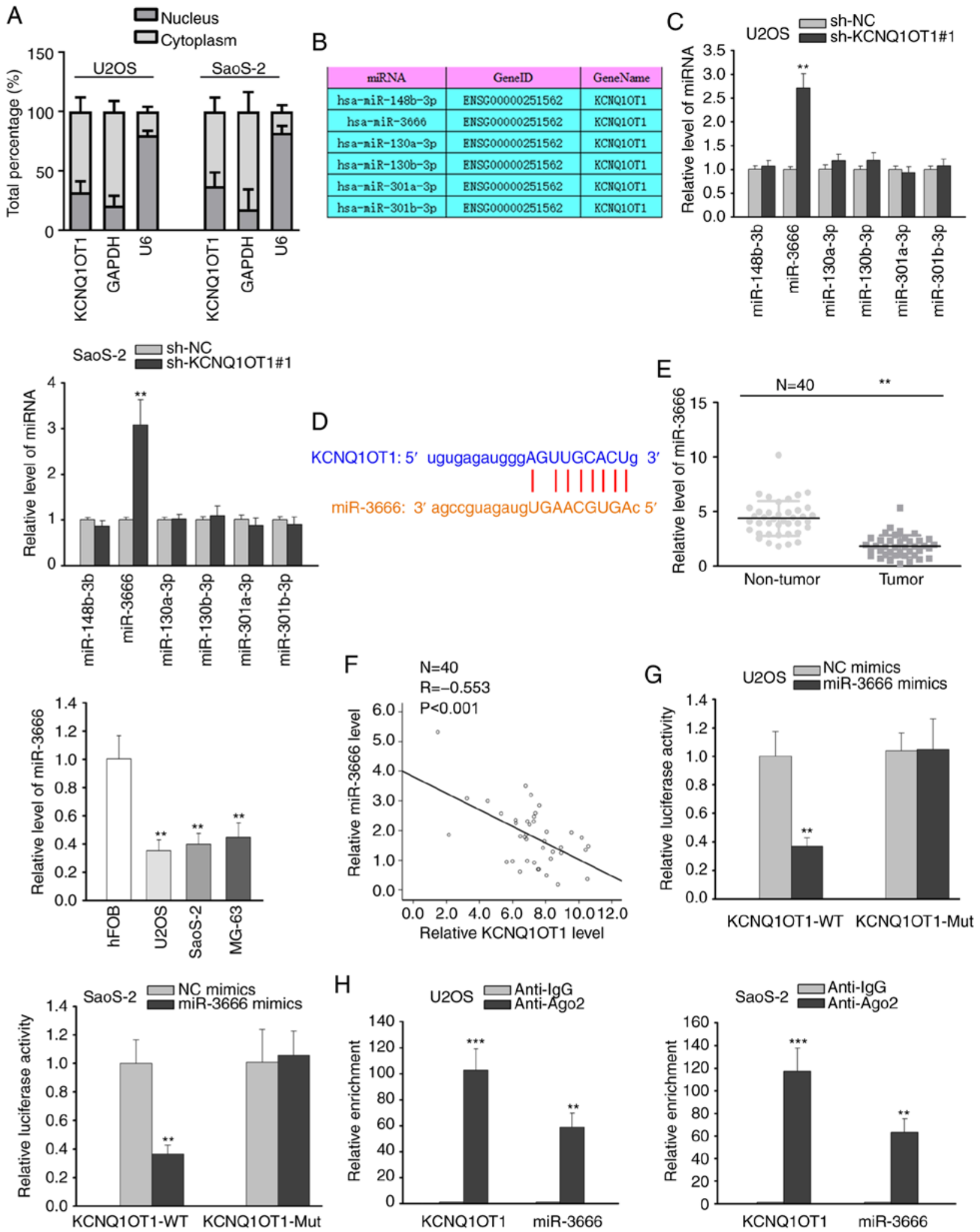

Figure 2. KCNQ1OT1 serves as a molecular sponge of miR-3666. (A) Nuclear-cytoplasmic fractionation displayed the subcellular distribution of KCNQ1OT1 in U2OS and SaoS-2 cells. (B) starBase predicted the potential miRNAs that might bind to KCNQ1OT1. (C) RT-qPCR exhibited expression levels of predicted miRNAs following transfection with sh-KCNQ1OT1\#1. (D) The predicted binding site between KCNQ1OT1 and miR-3666. (E) RT-qPCR was applied to detect miR-3666 expression in OS tissues and cell lines. Adjacent non-tumor tissues and osteoblast cell line were taken as control, separately. (F) Pearson's correlation analysis was utilized to determine the correlation between the expression of KCNQ1OT1 and miR-3666 in OS tissues. (G and H) Luciferase reporter and RIP assays both revealed the interaction between KCNQ1OT1 and miR-3666. ${ }^{* *} \mathrm{P}<0.01,{ }^{* * *} \mathrm{P}<0.001$ vs. respective control. OS, osteosarcoma.

that the upregulation of KLF4 counteracted the suppressive effects of KCNQ1OT1 silencing on Wnt/ $\beta$-catenin signaling in U2OS cells (Figs. 4F and S3). The above-mentioned data indicated that KCNQ1OT1 promoted cell proliferation, migration, invasion and Wnt $/ \beta$-catenin signaling in OS by targeting the miR-3666/KLF7 axis. 

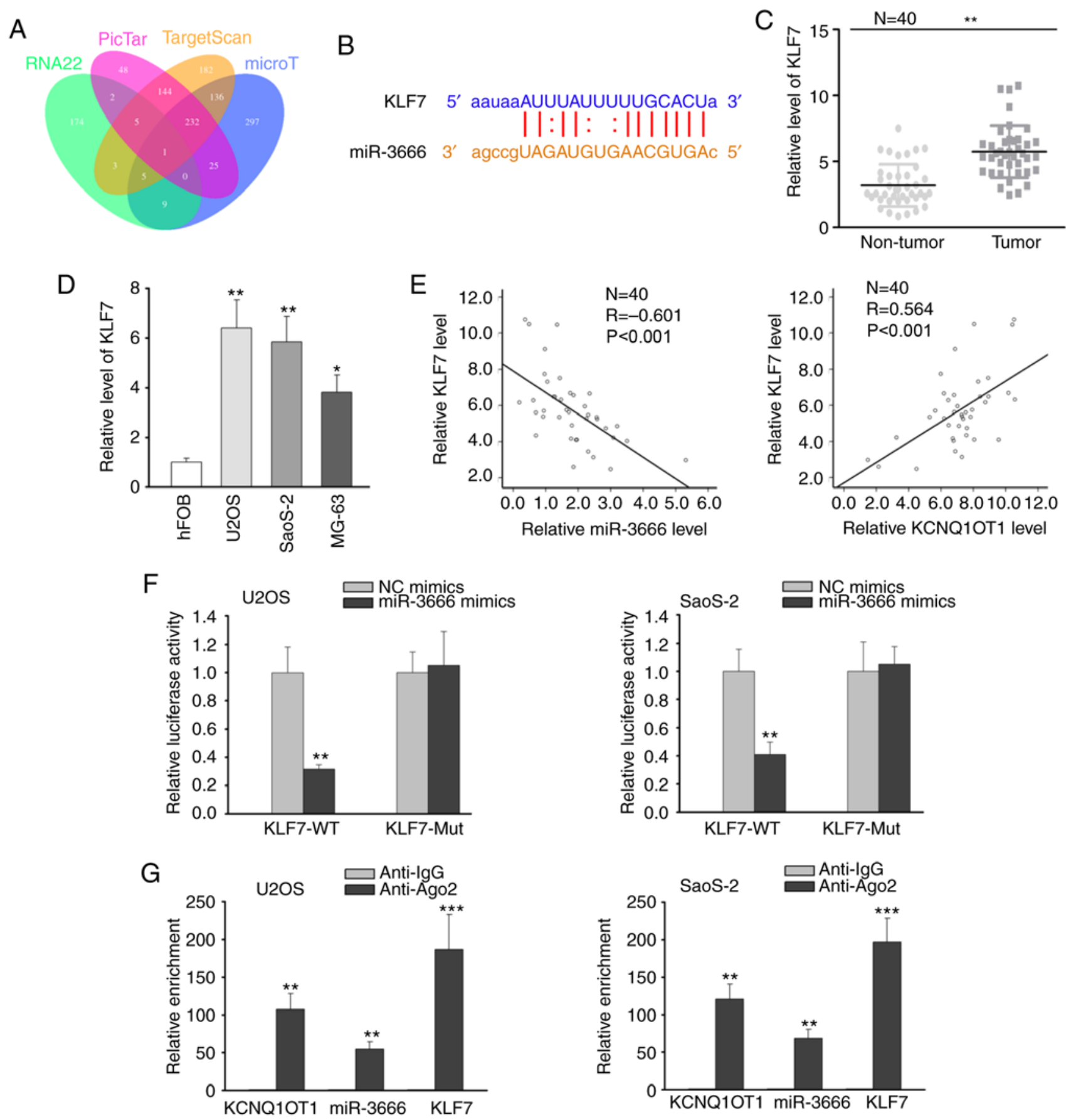

Figure 3. KLF7 is a downstream target of miR-3666. (A) The Venn diagram shows the candidate genes targeted by miR-3666. (B) The predicted binding sites of miR-3666 on KLF7 3'UTR. (C and D) KLF7 expression in OS tissues and cell lines was assessed by RT-qPCR analysis. (E) Expression association between KLF7 and miR-3666 or KCNQ1OT1 was explored by Pearson's correlation analysis. (F and G) The interaction between KLF7 and miR-3666 was verified by luciferase reporter and RIP assays. ${ }^{*} \mathrm{P}<0.05,{ }^{* *} \mathrm{P}<0.01,{ }^{* * *} \mathrm{P}<0.001$ vs. respective control. OS, osteosarcoma.

\section{Discussion}

In recent years, mounting evidence has indicated that IncRNAs play a role in the development of OS. For instance, IncRNA B4GALT1-AS1 has been shown to be upregulated in OS, and to promote OS cell stemness and migration (22). IncRNA SNHG4 has also been shown to facilitate tumor growth by sponging miR-224-3p and a high SNHG4 expression is associated with poor prognosis in OS (23). In the present study, KCNQ1OT1 was discovered to be highly expressed in OS tissues and cell lines. Patients with OS with a high KCNQ1OT1 expression exhibited a worse prognosis than those with a low KCNQ1OT1 expression. Moreover, the inhibition of KCNQ1OT1 suppressed cell proliferation, migration and invasion in OS. More importantly, KCNQ1OT1 silencing also inactivated $\mathrm{Wnt} / \beta$-catenin signaling. The results of the in vivo assay indicated that KCNQ1OT1 knockdown suppressed tumor growth in OS. All these data suggested that KCNQ1OT1 served as a tumor promoter in OS.

miRNAs belong to the cluster of non-coding RNAs which are 21-23 nucleotides in length and have been reported to play a key role in cancer progression $(24,25)$. It has been validated 

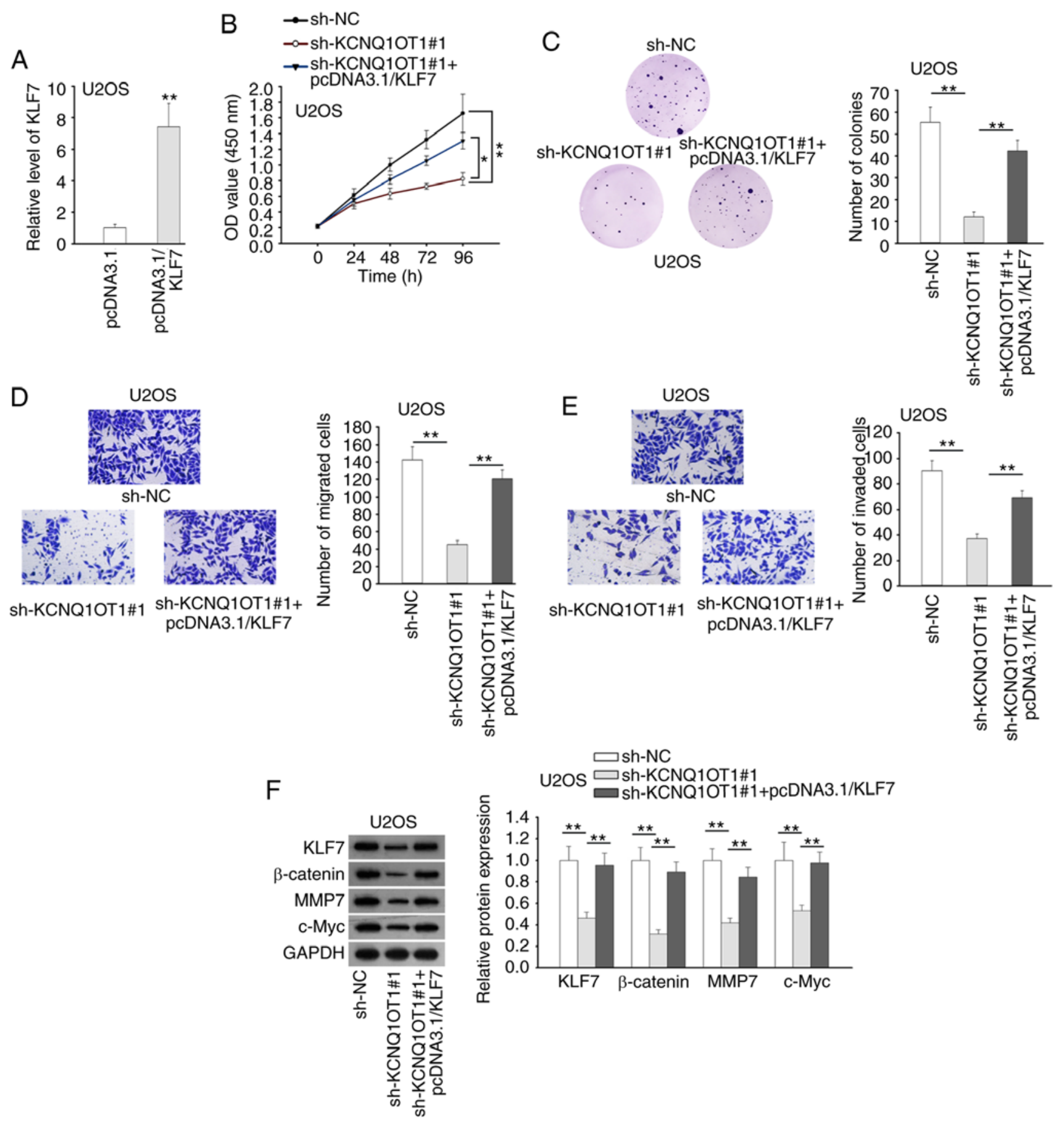

Figure 4. KCNQ1OT1 drives OS progression and activates Wnt/ $\beta$-catenin signaling via targeting the miR-3666/KLF7 axis. (A) Effect of pcDNA3.1/KLF7 on KLF7 expression was examined in U2OS cells. (B and C) Proliferation of U2OS cells transfected with indicated plasmids was evaluated by CCK-8 and colony formation assays. (D and E) Transwell assay presented cell migratory and invasive abilities of U2OS cells transfected with the indicated plasmids. (F) The protein levels of $\beta$-catenin, MMP7, KLF7 and c-Myc were assessed by western blot analysis in each group. ${ }^{*} \mathrm{P}<0.05,{ }^{* * *} \mathrm{P}<0.01$ vs. respective control. OS, osteosarcoma; KLF7, Kruppel-like factor 7; MMP7, matrix metalloproteinase 7.

that lncRNAs can serve as ceRNAs to increase target gene expression by competitively combining with miRNAs (26). The ceRNA network has been reported in diverse cancers, such as gastric cancer (27), bladder cancer (28), colorectal cancer (29) and OS (30). In the present study, miR-3666 was predicted to be a downstream miRNA of KCNQ1OT1. Previously, miR-3666 was proposed as a tumor suppressor in several types of cancer. miR-3666 was shown to suppress cell growth in lung cancer by targeting BPTF (31). miR-3666 was also shown to suppress the expression of SIRT7 to impair the growth of NSCLC cells (32). In the present study, miR-3666 expression was found to be markedly downregulated in OS tissues and cell lines. Moreover, there was a negative correlation between the expression of KCNQ1OT1 and that of miR-3666 in OS tissues. Furthermore, KCNQ1OT1 was corroborated to interact with miR-3666. Taken together, these findings indicate that KCNQ1OT1 serves as a sponge of miR-3666 in OS.

KLF7 has been widely reported in cancers and plays a critical role in cancer progression. For example, miR-185 has been 
shown to block cell proliferation and invasion in NSCLC cells by targeting KLF7 (33). LINC00668 facilitates cell motility in NSCLC cells by sponging miR-193a and increases the expression of KLF7 (34). In the present study, KLF7 expression was conspicuously upregulated in OS tissues and cell lines. Moreover, it was verified that KLF7 bound to miR-3666 in OS cells. Notably, the overexpression of KLF7 abolished the miR-3666 amplification-mediated suppression of OS cell proliferation, migration and invasion. Rescue assays manifested that the suppressed OS progression and Wnt/ $\beta$-catenin signaling induced by KCNQ1OT1 silencing were abrogated by KLF7 overexpression.

In conclusion, the present study demonstrates that KCNQ1OT1 predict an unfavorable prognosis, accelerates tumor growth and activates Wnt/ $\beta$-catenin signaling in OS by sponging miR-3666 and targeting KLF7, suggesting that KCNQ1OT1 is a promising prognostic biomarker in OS. The findings of this study may be aid in the investigation of potential treatment strategies for the patients with OS.

\section{Acknowledgements}

The authors acknowledge the support provided by the members of the laboratory of the Eighth Affiliated Hospital, Sun Yat-Sen University.

\section{Funding}

No funding was received.

\section{Availability of data and materials}

The datasets analyzed during the current study are available from the corresponding author on reasonable request.

\section{Authors' contributions}

$\mathrm{AH}, \mathrm{SJ}$ and $\mathrm{WH}$ were involved in the study design, data curation, formal analysis and methodology. AH, YW, SM, ZW, KL and QZ were involved in data curation and investigation. $\mathrm{AH}$, JZ, ZL and LC were responsible for supervising and tracking the progress of the experiments, and assisting in the smooth progress of the research. JZ, ZL and LC were also involved in the search for references required for the study. All authors agree that this is the final submission.

\section{Ethics approval and consent to participate}

The present study was approved by the Ethics Committee of the Eighth Affiliated Hospital, Sun Yat-Sen University. Written informed consent was obtained from all patients for the use of human clinical tissues in the present study.

\section{Patient consent for publication}

Not applicable.

\section{Competing interests}

The authors declare that they have no competing interests.

\section{References}

1. Smolle MA and Pichler M: The role of long non-coding RNAs in osteosarcoma. Noncoding RNA 4: 7, 2018.

2. Mirabello L, Troisi RJ and Savage SA: Osteosarcoma incidence and survival rates from 1973 to 2004: Data from the surveillance, epidemiology, and end results program. Cancer 115: 1531-1543, 2009.

3. Meyers PA, Schwartz CL, Krailo M, Kleinerman ES, Betcher D, Bernstein ML, Conrad E, Ferguson W, Gebhardt M, Goorin AM, et al: Osteosarcoma: A randomized, prospective trial of the addition of ifosfamide and/or muramyl tripeptide to cisplatin, doxorubicin, and high-dose methotrexate. J Clin Oncol 23: 2004-2011, 2005.

4. Bishop MW, Janeway KA and Gorlick R: Future directions in the treatment of osteosarcoma. Curr Opin Pediatr 28: 26-33, 2016.

5. Biazzo A and De Paolis M: Multidisciplinary approach to osteosarcoma. Acta Orthop Belg 82: 690-698, 2016.

6. Reed DR, Hayashi M, Wagner L, Binitie O, Steppan DA, Brohl AS, Shinohara ET, Bridge JA, Loeb DM, Borinstein SC and Isakoff MS: Treatment pathway of bone sarcoma in children, adolescents, and young adults. Cancer 123: 2206-2218, 2017.

7. Dean DC, Shen S, Hornicek FJ and Duan Z: From genomics to metabolomics: Emerging metastatic biomarkers in osteosarcoma. Cancer Metastasis Rev 37: 719-731, 2018.

8. Mercer TR, Dinger ME and Mattick JS: Long non-coding RNAs: Insights into functions. Nat Rev Genet 10: 155-159, 2009

9. Huarte M: The emerging role of IncRNAs in cancer. Nat Med 21: 1253-1261, 2015.

10. Guo X, Wei Y, Wang Z, Liu W, Yang Y, Yu X and He J: LncRNA LINC00163 upregulation suppresses lung cancer development though transcriptionally increasing TCF21 expression. Am J Cancer Res 8: 2494-2506, 2018.

11. Jing $\mathrm{H}$, Xia $\mathrm{H}$, Qian $\mathrm{M}$ and $\mathrm{Lv} \mathrm{X}$ : Long noncoding RNA CRNDE promotes non-small cell lung cancer progression via sponging microRNA-338-3p. Biomed Pharmacother 110: 825-833, 2019.

12. Chen Z, Hu X, Wu Y, Cong L, He X, Lu J, Feng J and Liu D: Long non-coding RNA XIST promotes the development of esophageal cancer by sponging miR-494 to regulate CDK6 expression. Biomed Pharmacother 109: 2228-2236, 2019.

13. Bian Y, Gao G, Zhang Q, Qian H, Yu L, Yao N, Qian J, Liu B and Qian X: KCNQ1OT1/miR-217/ZEB1 feedback loop facilitates cell migration and epithelial-mesenchymal transition in colorectal cancer. Cancer Biol Ther 20: 886-896, 2019.

14. Dong Z, Yang P, Qiu X, Liang S, Guan B, Yang H, Li F, Sun L, Liu H, Zou G and Zhao K: KCNQ1OT1 facilitates progression of non-small-cell lung carcinoma via modulating miRNA-27b-3p/HSP90AA1 axis. J Cell Physiol 234: 11304-11314, 2019.

15. Li Y, Li C, Li D, Yang L, Jin J and Zhang B: lncRNA KCNQ1OT1 enhances the chemoresistance of oxaliplatin in colon cancer by targeting the miR-34a/ATG4B pathway. Onco Targets Ther 12: 2649-2660, 2019.

16. Wei R, Ding C, Rodriguez RA and Del Mar Requena Mullor M: The SOX2OT/miR-194-5p axis regulates cell proliferation and mobility of gastric cancer through suppressing epithelialmesenchymal transition. Oncol Lett 16: 6361-6368, 2018.

17. Luo Y, Tan W, Jia W, Liu Z, Ye P, Fu Z, Lu F, Xiang W, Tang L, Yao L, et al: The long non-coding RNA LINC01606 contributes to the metastasis and invasion of human gastric cancer and is associated with Wnt/ $\beta$-catenin signaling. Int J Biochem Cell Biol 103: 125-134, 2018.

18. Livak KJ and Schmittgen TD: Analysis of relative gene expression data using real-time quantitative PCR and the 2(-Delta Delta C(T)) method. Methods 25: 402-408, 2001.

19. Gao X, Ge J, Li W, Zhou W and Xu L: LncRNA KCNQ1OT1 promotes osteogenic differentiation to relieve osteolysis via Wnt/ $\beta$-catenin activation. Cell Biosci 8: 19, 2018.

20. Tay Y, Rinn J and Pandolfi PP: The multilayered complexity of ceRNA crosstalk and competition. Nature 505: 344-352, 2014.

21. Karreth FA and Pandolfi PP: ceRNA cross-talk in cancer: When ce-bling rivalries go awry. Cancer Discov 3: 1113-1121, 2013.

22. Li Z, Wang Y, Hu R, Xu R and Xu W: LncRNA B4GALT1-AS1 recruits HuR to promote osteosarcoma cells stemness and migration via enhancing YAP transcriptional activity. Cell Prolif 51: e12504, 2018. 
23. Xu R, Feng F, Yu X, Liu Z and Lao L: LncRNA SNHG4 promotes tumour growth by sponging miR-224-3p and predicts poor survival and recurrence in human osteosarcoma. Cell Prolif 51: e12515, 2018.

24. Srivastava SK, Bhardwaj A, Leavesley SJ, Grizzle WE, Singh S and Singh AP: MicroRNAs as potential clinical biomarkers: Emerging approaches for their detection. Biotech Histochem 88: 373-387, 2013.

25. Adams BD, Parsons C, Walker L, Zhang WC and Slack FJ: Targeting noncoding RNAs in disease. J Clin Invest 127: 761-771, 2017.

26. Kopp F and Mendell JT: Functional classification and experimental dissection of long noncoding RNAs. Cell 172: 393-407, 2018.

27. Yang XZ, Cheng TT, He QJ, Lei ZY, Chi J, Tang Z, Liao QX, Zhang H, Zeng LS and Cui SZ: LINC01133 as ceRNA inhibits gastric cancer progression by sponging miR-106a-3p to regulate APC expression and the Wnt/ $\beta$-catenin pathway. Mol Cancer 17: 126, 2018.

28. Zhan Y, Chen Z, Li Y, He A, He S, Gong Y, Li X and Zhou L: Long non-coding RNA DANCR promotes malignant phenotypes of bladder cancer cells by modulating the miR-149/MSI2 axis as a ceRNA. J Exp Clin Cancer Res 37: 273, 2018.
29. Liu B, Pan S, Xiao Y, Liu Q, Xu J and Jia L: LINC01296/ miR-26a/GALNT3 axis contributes to colorectal cancer progression by regulating O-glycosylated MUC1 via PI3K/AKT pathway. J Exp Clin Cancer Res 37: 316, 2018.

30. Fei D, Zhang X, Liu J, Tan L, Xing J, Zhao D and Zhang Y: Long noncoding RNA FER1L4 suppresses tumorigenesis by regulating the expression of PTEN targeting miR-18a-5p in osteosarcoma. Cell Physiol Biochem 51: 1364-1375, 2018.

31. Pan L, Tang Z, Pan L and Tang R: MicroRNA-3666 inhibits lung cancer cell proliferation, migration, and invasiveness by targeting BPTF. Biochem Cell Biol 97: 415-422, 2018.

32. Shi H, Ji Y, Zhang D, Liu Y and Fang P: MicroRNA-3666-induced suppression of SIRT7 inhibits the growth of non-small cell lung cancer cells. Oncol Rep 36: 3051-3057, 2016.

33. Zhao L, Zhang Y, Liu J, Yin W, Jin D, Wang D and Zhang W: MiR-185 inhibits cell proliferation and invasion of non-small cell lung cancer by targeting KLF7. Oncol Res 27: 1015-1023, 2019.

34. An YX, Shang YJ, Xu ZW, Zhang QC, Wang Z, Xuan WX and Zhang XJ: STAT3-induced long noncoding RNA LINC00668 promotes migration and invasion of non-small cell lung cancer via the miR-193a/KLF7 axis. Biomed Pharmacother 116: 109023, 2019. 